\title{
New Tracking System of Human Scapula Using Ultrasonography and Motion Sensors: A Descriptive Test-Retest Design
}

\author{
Manuel Trinidad-Fernández, Manuel González-Sánchez, and Antonio I. Cuesta-Vargas
}

\begin{abstract}
Context: Several studies have shown that the kinematics of the scapula is altered in many disorders that affect the shoulder. Description of scapular motion in the chest continues to be a scientific and clinical challenge. Objective: To check the validity and reliability of a new, minimally invasive method of tracking the internal and external rotation of the scapula using ultrasound imaging combined with the signal provided by a 3-dimensional electromagnetic sensor. Design: A cross-sectional study with a repeated-measures descriptive test-retest design was employed to evaluate this new tracking method. The new method was validated in vitro and the reliability of data over repeated measures between scapula positions was calculated in vivo. Setting: University laboratory. Participants: A total of 30 healthy men and women. Main Outcome Measure: The validation of the scapula rotation tracking using the in vitro model was calculated by Pearson correlation test between a 2-dimensional crosscorrelation algorithm of the new method and another software image. The reliability of the tracking of the scapula rotation was measured using the intraclass correlation coefficient. Results: In the validation in vitro, the correlation of rotations obtained by the 2 methods was good $(r=.77, P=.01)$. The reliability in vivo had excellent results (intraclass correlation coefficient $=.88 ; 95 \%$ confidence interval, .82-.93) in the test-retest analysis of 8 measures. The intrarater analysis of variance test showed no significant differences between the measures $(P=.85, F=0.46)$. Conclusion: Ultrasound imaging combined with a motion sensor to track the scapula has been shown to be a reliable and valid method for measuring internal and external rotation during separation of the upper limb.
\end{abstract}

Keywords: tracking motion, kinematics, scapulothoracic joint

The description of scapular motion in the thorax continues to be a scientific and clinical challenge because the kinematics of the scapula is altered in many shoulder disorders. ${ }^{1,2}$ Scapular protraction is defined as scapular anterior tilting together with scapular internal rotation. ${ }^{3}$

The many methods available to track the movement of the scapula can be divided into invasive and noninvasive methods. Noninvasive methods, such as motion sensors on the skin, can be inaccurate because of the deformation and movement of the overlying skin. ${ }^{4,5}$ Alternative and more accurate invasive methods for measuring scapular movement, such as transcortical pins are available, but their greatest problem is that they cannot be used in clinical practice. ${ }^{1}$ A method which is both accessible and accurate is needed to determine a valid measure of scapular movement. The incorporation of imaging processing to understand the movement inside the body with a coordinate transform system to set the scapula in space could provide useful insights. We validated a new method, which combined ultrasound imaging with the signal provided by a 3-dimensional electromagnetic sensor in an anatomical model. ${ }^{6}$ This appears to be the first use of this methodology in humans.

This study aimed to evaluate a new, minimally invasive method of tracking internal and external rotation of the scapula using ultrasound imaging combined with the signal provided by a

\footnotetext{
Trinidad-Fernández, González-Sánchez, and Cuesta-Vargas are with the Departamento de Fisioterapia, Instituto de Biomedicina de Malaga (IBIMA), Grupo de Clinimetria (AE-14), Universidad de Málaga, Andalucía Tech, Facultad de Ciencias de la Salud, Malaga, Spain. Cuesta-Vargas is also with the School of Clinical Sciences, Faculty of Health, Queensland University of Technology, Brisbane, QLD, Australia. Cuesta-Vargas (acuesta@uma.es) is corresponding author.
}

3-dimensional electromagnetic sensor and to assess the reliability of data over repeated measures between scapula positions using this new method in humans.

\section{Methods}

\section{Study Design}

A cross-sectional study with a repeated-measures descriptive testretest design was employed for the evaluation of this new tracking method.

\section{Participants}

Young adults (18-30 y old), who were asymptomatic and not performing more than 2 hours of physical exercise per week were selected. Exclusion criteria: had undergone a surgical intervention or had suffered a trauma/fracture in the upper limb in the year prior to the study, suffered a chronic problem in the neuromusculoskeletal system, had a job that required repetitive use of the upper limb so that the scapular kinematics could be altered, had a body mass index greater than $30 \mathrm{~kg} / \mathrm{m}^{2}$, or were pregnant. Ethical approval was obtained from University of Malaga, and informed consent was obtained from each participant. The current study was developed respecting the principles of the Declaration of Helsinki.

\section{Setup and Sensor Tracking of Ultrasound Transducer}

MyLab ${ }^{\mathrm{TM}} 25$ Gold (ESAOTE, Genoa, Italy), a 2-dimensional ultrasound equipped with a linear array transducer, was used for the 


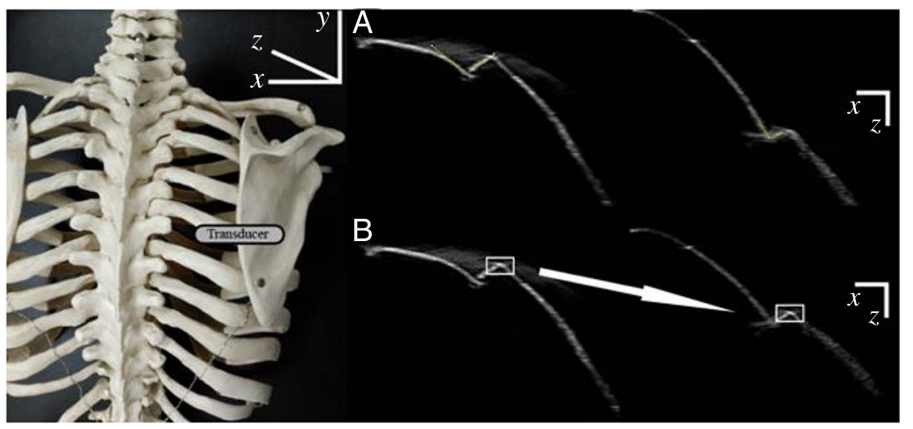

Figure 1 - References system in scapula and processing of the ultrasonography images. (A) With ImageJ, we marked the angle between the rib and the medial border of the scapula in 2 different positions. (B) We selected the medial border of the scapula in the first position in order to perform the cross-correlation. The algorithm from MATLAB searched the second image for the position the same anatomical structure. The crosscorrelation gave the rotation angle of the scapula between the 2 images.

images and a Polhemus FASTRAK (Colchester, VT) recorded electromagnetic sensor positions. This system records sensor position and movement relative to a source transmitter with 6 degrees of freedom. The sensor was placed on the ultrasound transducer to $3.5 \mathrm{~cm}$ with adhesive tape to avoid interference. This new ultrasound-based tracking motion allowed us to record the position and orientation of one coordinate system relative to a global reference system.

\section{Imaging Processing}

The rotation was calculated with ImageJ 1.48v (Bethesda, MD) (regarded as the gold standard) using the difference of the angles formed between the rib and the medial border of the scapula (Figure 1a).

MATLAB (Natick, MA) 2014 software was used to determine the rotation of the scapula, with a 2-dimensional crosscorrelation algorithm to observe the degrees of rotation between a pair of selected images in B-mode. Therefore, 2 positions of the scapula were chosen for subsequent analysis (Figure 1b). The 2-dimensional cross-correlation of an M-by-N matrix $\mathrm{A}$ and an M-by-N matrix $\mathrm{B}$ was given by:

$$
C(i, j)=\sum_{m=0}^{M_{a}-1} \sum_{n=0}^{N_{a}-1} A(m, n) \cdot \operatorname{conj}[B(m+i, n+j)],
$$

where, $0 \leq i \leq M_{a}+M_{b}-1$ and $0 \leq j \leq N_{a}+N_{b}-1$.

A window showing the medial border of the scapula was selected from the first ultrasound images collected in the first position as a reference. The tracking algorithm was applied to find the region most similar to the references in subsequent image frames. The ultrasound images and electromagnetic results were synchronized for off-line analysis.

The transducer was placed on the right scapula benchmark above the sixth rib.

\section{Coordinate System Transformations}

Coordinate system transformations describe the position and orientation of one coordinate system relative to a reference system by a $4 \times 4$ matrix. The overall coordinate system transformations is obtained from the matrix:

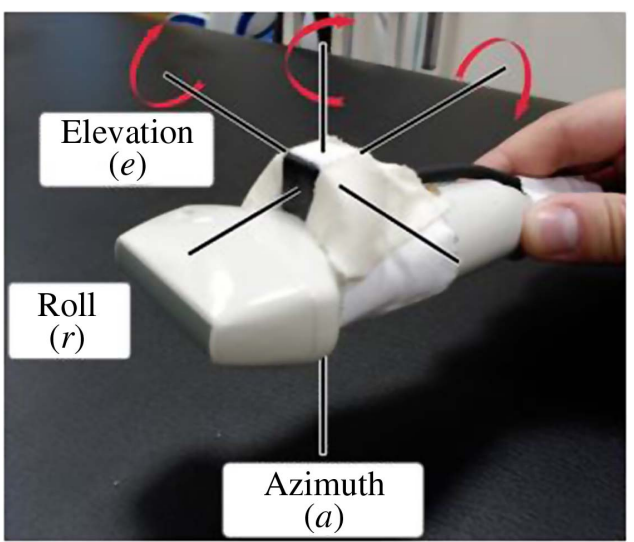

Figure 2 - Representation of the 3 Euler angle displacements in the transductor.

$C(x, y, z, a, e, r)=$

$[\cos a \cos e \cos a \sin e \sin r-\sin a \cos r \cos a \sin e \cos r+\sin a \sin r x]$ $\sin a \cos e \sin a \sin e \sin r+\cos a \cos r \sin a \sin e \cos r-\cos a \cos r y$ $\left.\begin{array}{cccc}-\sin e & \cos e \sin r & \cos e \cos r & z \\ 0 & 0 & 0 & 1\end{array}\right]$,

where $x, y$, and $z$ are 3 linear offsets and $a, e$, and $r$ are 3 Euler angle displacements: azimuth, elevation, and roll, respectively (Figure 2). The Euler angles define a strict rotation sequence: first about the $z$-axis, then about the $y$-axis, and then about the $x$-axis of the displaced coordinate system.

\section{In Vitro Validation Experiment}

An anatomical model of a thorax was immersed in a water container in order to produce a simulated ultrasonography image of a human thorax. The scapula was fixed with a screw in 2 interchangeable positions. The scapula was positioned at an angle of $20^{\circ}$ and $25^{\circ}$ between the spinous processes of the vertebrae and the lower angle of the scapula. The water container was filled until it covered the ribs and the scapula, with an additional $1 \mathrm{~cm}$ of water added to help the ultrasound registration. The transducer head was in contact with water and fixed on an articulated arm to prevent movement.

Ten ultrasound images were recorded in 2 different scapula positions. The transducer position was different between the positions. Images were accompanied by sensor information. The images were analyzed offline using ImageJ and MATLAB.

\section{In Vivo Reliability Experiment}

With the subjects in a standing position, an experienced physiotherapist marked the skin after palpating the scapula and the sixth rib.

The 2 different scapula positions of the subjects were $0^{\circ}$ and $120^{\circ}$ of shoulder abduction in the frontal plane (Figure 3 ). The subject placed their upper limb on a shelf that prevented any change in the abduction angle arm due to fatigue. The transducer was fixed to an articulated arm.

On the same day, 8 images per position were recorded, not interleaved, with sensor information. The transducer changed position due to the movement of the scapula along the ribcage. 


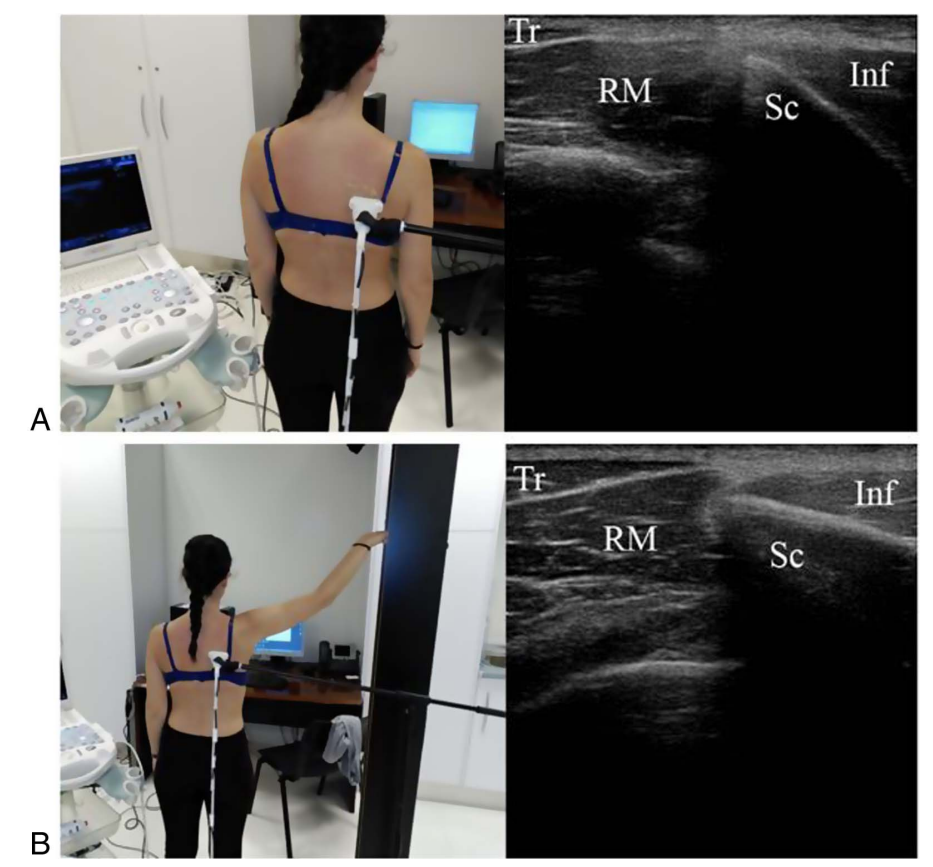

Figure 3 - Acquisition of data of human measurements: (A) standard arm position and (B) $120^{\circ}$ shoulder abduction.

\section{Data Analysis}

A composite measure of the scapula rotation for each repetition was calculated from the raw data plus the sensor result and used for subsequent analysis.

A Pearson correlation test for related variables was performed. Results of the correlation were classified: poor $(\leq .49)$, moderate (.50-.74), and strong ( $\geq .75) .^{7}$

Intratester reliability analysis using an intraclass correlation coefficient 2-way random-effects model 2.1 (ICC) from a repeatedmeasures analysis of variance test was performed to determine the reliability of the 8 measures. ICC classification: good, .61 to .80; excellent, .81 to $1.00 .^{8}$

Descriptive anthropometric variables of the sample were calculated. The mean of the results was shown with the SEM. All data were analyzed using SPSS (version 15; Armonk, NY), where $P<.05$ was considered statistically significant.

\section{Results}

Data were collected from 30 subjects (15 females; average age: 21.87 [1.73] y, average body mass index: 22.15 [2.52] kg/m²).

\section{External Validity}

The mean total rotation was $12.32^{\circ}\left(0.29^{\circ}\right)(\mathrm{SEM}=0.09)$ using ImageJ and $12.66^{\circ}\left(1.10^{\circ}\right)(\mathrm{SEM}=0.34)$ using MATLAB. The correlation of rotations obtained by the 2 software methods was $r=.77$ with a significance level of $P=.01$. The error of the 2-dimensional correlation obtained using MATLAB had an average of $0.86 \%(0.02 \%)$.

\section{In Vivo Reliability}

The means of the rotations measured during the in vivo phase were from $2.37^{\circ}\left(7.71^{\circ}\right)$ to $3.59^{\circ}\left(8.53^{\circ}\right)(\mathrm{SEM}=1.35-1.72)$. In the test-retest analysis of 8 measures, reliability was $\mathrm{ICC}=.88$
(95\% confidence interval, .82-.93). The intrarater analysis of variance test showed no significant differences between the measures $(P=.85, F=0.46)$.

\section{Discussion}

The aim was achieved, and the new method has demonstrated very good validity and reliability in tracking the internal and external rotation of the scapula.

This study presents a new strategy to overcome the problem of scapula tracking with a method that is easy to use in clinical practice and does not suffer from the problems of skin interference. There is a lot of information in the literature about the kinematics of the scapula, but all methods reported require the sensor to be fixed on the skin or the bone. ${ }^{9}$ This is the first time that the scapula is tracked by adding the ultrasound image from the inside to the information obtained from the movement of the transductor on the outside. The image processing and positioning used in this study also offers the possibility of dynamic analysis.

The level of reliability observed using this methodology was excellent $^{8}(\mathrm{ICC}=.88 ; 95 \%$ confidence interval, .82-.93). A previous study that used a similar methodology on the lumbar spine 6 also presented excellent reliability values, although with slightly higher quantitative data than the present study $($ ICC $=.996-.999){ }^{6}$ The different type of tissues found in these regions (shoulderlumbar) and the structures analyzed could explain the small differences found between the 2 studies. ${ }^{10}$

This study has several limitations. The ultrasound shows results in 2 dimensions, which forces us to discard 1 of the 3 dimensions. In addition, the amplitude of the ultrasound image is limited to the size of the ultrasound head. Finally, subjects with different characteristics in their connective tissue may have different echogenicity. ${ }^{10}$

Future studies should continue to describe the clinical implications of this new method.

\section{Acknowledgments}

The authors would like to thank all the participants. They would also like to thank Mariano Tarifa for his support in MATLAB codes, and Adrian G. Jorge and Ruben Nieto for improving those codes. The authors have no conflicts of interest to disclose.

\section{References}

1. Roren A, Lefevre-Colau MM, Poiraudeau S, Fayad F, Pasqui V, Roby-Brami A. A new description of scapulothoracic motion during arm movements in healthy subjects. Man Ther. 2015;20(1):46-55. PubMed ID: 25034959 doi:10.1016/j.math.2014.06.006

2. Ludewig PM, Reynolds JF. The association of scapular kinematics and glenohumeral joint pathologies. J Orthop Sports Phys Ther. 2009; 39(2):90-104. PubMed ID: 19194022 doi:10.2519/jospt.2009.2808

3. Struyf F, Meeus M, Fransen E, et al. Interrater and intrarater reliability of the pectoralis minor muscle length measurement in subjects with and without shoulder impingement symptoms. Man Ther. 2014; 19(4):294-298. PubMed ID: 24815592 doi:10.1016/j.math.2014. 04.005

4. Lovern B, Stroud LA, Evans RO, Evans SL, Holt CA. Dynamic tracking of the scapula using skin-mounted markers. Proc Inst Mech Eng H. 2009;223(7):823-831. PubMed ID: 19908421 doi:10.1243/ 09544119JEIM554 
5. Morgado Ramírez DZ, Strike S, Lee RY. Measurement of transmission of vibration through the human spine using skin-mounted inertial sensors. Med Eng Phys. 2013;35(5):690-695. doi:10.1016/ j.medengphy.2012.12.013

6. Cuesta-Vargas AI. Development of a new ultrasound-based system for tracking motion of the human lumbar spine: reliability, stability and repeatability during forward bending movement trials. Ultrasound Med Biol. 2015;41(7):2049-2056. PubMed ID: 25864018 doi:10.1016/j.ultrasmedbio.2015.02.015

7. Field A. Discovering Statistics Using IBM SPSS Statistics. 4th ed. Thousand Oaks, CA: SAGE Publications Ltd; 2013.
8. Shrout PE, Fleiss JL. Intraclass correlations: uses in assessing rater reliability. Psychol Bull. 1979;86(2):420-428. PubMed ID: 18839484 doi:10.1037/0033-2909.86.2.420

9. Keshavarz R, Bashardoust Tajali S, Mir SM, Ashrafi H. The role of scapular kinematics in patients with different shoulder musculoskeletal disorders: a systematic review approach. J Bodyw Mov Ther. 2017;21(2): 386-400. PubMed ID: 28532884 doi:10.1016/j.jbmt.2016.09.002

10. Langevin HM, Stevens-Tuttle D, Fox JR, et al. Ultrasound evidence of altered lumbar connective tissue structure in human subjects with chronic low back pain. BMC Musculoskelet Disord. 2009;10(1):151. doi:10.1186/1471-2474-10-151 